\title{
A Novel Missense Variant in the ESRRB Gene Causing Nonsyndromic Hearing Loss: In Silico Analyses of a Case
}

\section{Tohid Ghasemnejad}

Tabriz University of Medical Sciences

Mahmoud Shekari Khaniani

Tabriz University of Medical Sciences

Jafar Nouri Nojadeh

Tabriz University of Medical Sciences

Sima Mansoori Derakhshan ( $\square$ simansooris@gmail.com )

Tabriz University of Medical Sciences

\section{Research Article}

Keywords: Hearing loss, consanguineous marriage, NGS, ESRRB

Posted Date: July 15th, 2021

DOI: https://doi.org/10.21203/rs.3.rs-698699/v1

License: (c) (i) This work is licensed under a Creative Commons Attribution 4.0 International License. Read Full License 


\section{Abstract}

Background: Genetic hearing loss (GHL) is a common heterogeneous disorder that can affect all ages, ethnicities, and genders. The most common form of hearing loss ( $\mathrm{HL})$ is autosomal recessive nonsyndromic hearing loss (ARNSHL) and in most cases specific genotype-phenotype correlation is indistinguishable. This study aimed to identify the genetic cause of hearing loss in an Iranian Azeri Turkish ethnicity family with consanguine marriage which is negative for GJB2, GJB6 and mitochondrially encoded 12S RRNA (MT-RNR1) deleterious mutations.

Methods: Targeted genome sequencing was applied for the detection of possible genetic causes of $\mathrm{HL}$ in this family. Co-segregation and in silico analysis of variant was performed by standard procedure.

Results: A missense variant, c.499G>A, was identified in the ESRRB gene. Healthy and affected members of the family confirmed co-segregation of the variant with ARNSHL in the pedigree and then the pathogenicity of the variant was confirmed by in silico analysis and ACMG Guidelines.

Conclusion: We report a novel missense variant in the ESRRB gene which seems to be a pathogenic variant. The result of this study suggests that the genetic background of hearing loss patients plays important role in the pathogenicity; moreover, targeted genomic capture is a powerful method that can discover pathogenic variants in heterogeneous disorders.

\section{Background}

One of the hereditary disorders that are considered a public health concern is hereditary hearing loss $(\mathrm{HHL})$ which is estimated to affect 1 in 650-1000 newborns in developed countries [1]. Although environmental agents can cause $\mathrm{HL}$, genetic factors have a significant contribution to its etiology [2]. Current knowledge shows that approximately $70 \%$ of all genetic HLs are in the form of non-syndromic, in which $\mathrm{HL}$ is the only clinical symptom, and $30 \%$ accounts for syndromic forms that hearing loss can be part of other syndromes [3]. HHL can be transmitted as autosomal dominant (20\%), autosomal recessive (70-80), X-linked (2-5\%), and mitochondrial (1\%) inheritance [4]. Based on variation databases including Human Gene Mutation Database (HGMD), ClinVar, and Single-Nucleotide Polymorphism database (dbSNP), more than 2000 variants have been determined in NSHL, in which, homozygous or compound heterozygous variants in the GJB2 gene and large deletions in the GJB6 gene are a common cause of hearing loss in Iran and most of the other countries [5-7]. Furthermore, previous researches have revealed that mutations in SLC26A4 [8] is another common cause of NSHL followed by MY015A [9] MY07A [7], CDH23 [10], PCDH15 [10], TECTA [11], PVK [12], TMC1 [13], LRTOMT [14], ILDR1 [15], MARVELD2 [7], OTOF [7], RDX [7], LOXHD1 [16], COL11A2 [17]. According to Bazazzadegan. et al study In Iran, the prevalence of the GJB2 mutation is variable, based on geographical location and ethnicity, and for example, in In Azerbaijan province (northwest of Iran where our study is done here with Azeri Turkish ethnicity), GJB2 mutations accounts for $22 \%$ of hearing losses; whereas, in Sistan and Baluchestan province (southeast of Iran with Baluch ethnicity) this amount is decreased to $8 \%[7]$. Since NSHL is a 
highly heterogeneous disorder and due to limited phenotypic variability genetic diagnosis is particularly challenging [18]. In recent years, significant advances in diagnosis method such as next-generation sequencing (NGS) have opened a new window for the heterogenic disorder; however, the production of thousands of variants identified [17] per individual is the challenge in NGS technology for heterogenic disorders; thus, correct interpretation, as well as ethnic-specific filtering by allele frequency is crucial when test results are used to direct clinical care $[19,20]$. In this light, this study aimed to establish targeted sequencing of some known and candidate $\mathrm{HL}$-causing genes in proband of a family of Iranian Azeri Turkish ethnicity with consanguine marriage, which are negative for GJB2,GJB6 and MT-RNR1 (A1555G) deleterious mutations.

\section{Methods}

\section{Patients}

An Iranian affected individual of Azeri Turkish ethnicity, whose parents had consanguineous marriage was recruited from Ebnsina Medical Genetic Laboratory of Tabriz. Physical examinations and audiological evaluation, and then clinical questionnaires on age, exposure to environmental factors, and the history of other diseases had been administered when tested for GJB2, GJB6 and MT-RNR1 (A1555G) mutations. Full informed consent was obtained from the patients and all members of their families which participated in the study. The study and methods used for the investigation involving humans were approved by the ethics committee of Tabriz University of Medical Sciences.

\section{Targeted genomic capturing and next-generation sequencing}

The experiment of targeted capturing was performed to screen possible mutations according to the Beijing Genomics Institute (BGI) protocols. A sequencing test was conducted using a custom-designed Nimblegen chip capturing the targeted genes followed by NGS. The test platform tested $>95 \%$ of all exons of the 89 candidate genes with a sensitivity of $>99 \%$. Point mutation, micro-insertion, deletion, and duplication ( $<20 \mathrm{bp}$ ) can be altogether detected. Bioinformatics analysis of the sequencing results using international mutation and polymorphism databases as well as BGI self-developed local database provides an association of the mutations/variations with the clinical conditions.

\section{Co-segregation analysis}

To evaluate the co-segregation of the variant with the phenotype, primers surrounding the damaging variant were designed by Primer3 software which includes forward: GCCACCAGGCTAAATCCTCTG and reverse: TTTCCAAGGTCACCAGGCAGT. After amplification by Eppendorf instrument and SinaClon PCR Master Mix kit (Cat. No.: MM2011), Sanger sequencing was performed by big dye kit (Cat. No.: 4337455) and Applied Biosystems (3130xl Genetic Analyzer) instrument, and finally chromas software 2.6.4 version was used to observe the obtained sequence.

\section{Evaluation of variants in the control population}


After obtaining informed consent from 200 healthy individuals of the same origin (Iranian Azeri Turkish), genomic DNA was extracted from peripheral blood leukocytes according to the salting out procedure, then by using primers flanking, the candidate gene PCR amplification and Sanger sequencing were performed with standard procedure.

\section{In silico analysis}

\section{Pathogenicity prediction of the variation}

For determination of the pathogenicity of identified variant, multiple bioinformatics software and web servers including Polyphen(http://genetics.bwh.harvard.edu/pph2/), SIFT(http://sift.jcvc.org/), FATHMM (http://fathmm.biocompute.org.uk/), MetaSVM (https://omictools.com/metasvmtool)

LRT(http://www.genetics.wustl.edu/jflab/lrt_query.html),

MutationAssessor(http://mutationassessor.org/r3/), MutationTastr (http://www.mutationtaster.org/),

LOFtool (http://www.ensembl.info/ecode/loftool/) GERP++

(http://mendel.stanford.edu/SidowLab/downloads/gerp/ ) were used.

\section{Protein conservation analysis}

For conservation analysis, the protein sequences were aligned to evaluate conservation and evolutionary relationships among vertebrate species by using the UniProtKB/UniRef100 server (http://www.uniprot.org/uniref/UniRef100).

\section{Molecular modeling}

The effect of missense mutations on the molecular level was analyzed by modeling software Yet Another Scientific Artificial Reality Application (YASARA) (http://www.yasara.org/ ). For ESRRB protein, the model was according to the PDB file with ID: 1 lo1.

\section{Protein stability}

The impact of amino acid substitutions on ESRRB protein structure stability was analyzed, by using MAESTRO (https://biwww.che.sbg.ac.at/maestro/web/maestro/workflow) web and SDM (Site Directed Mutator) (http://www structure.bioc.cam.ac.uk/sdm2) web servers.

\section{Population databases analysis}

Minor allele frequency (MAF) was used to discriminate common polymorphism from rare variants in the population database and the MAF $<1 \%$ was enriched for the functional mutation [5]. Population databases include 1000 Genome project(http://www.1000genomes.org/), Exome Sequencing Project (http://evs.gs.washington.edu/EVS/), and Exome Aggregation Consortium (http://exac.broadinstitute.org) which are databases excluded from the genetic variants previously reported as polymorphism.

\section{Results}




\section{Clinical evaluations}

In this family, both parents had normal hearing. The proband (IV.3) was a 3-year-old boy and the audiogram revealed bilateral, severe to profound HL (Figure 1). As stated by parents, hearing loss was congenital. No other abnormality indicating syndromic hearing loss was present. As much as remembered by the mother, there was no exposure to environmental factors and no special disease which would have made her use a particular medicine or antibiotic such as aminoglycoside.

\section{Molecular evaluation}

Targeted genomic capturing in this family indicated novel homozygous variation c.499G>A (p. Gly167Arg) in ESRRB gene (Estrogen Related Receptor Beta- NM_004452) with NCBI ss-2137543931 number which submitted by our group. The variant co-segregated with the ARNSHL phenotype (Figure 1) and was absent in 200 control populations (data have not shown). The result of mutagenicity assessment of c.499G $>A$ variation was predicted as a damaging variant by all databases without frequency in population databases studies (Table 1). Conservation evaluation of glycine in the 167th amino acid position of estrogen-related to receptor beta indicates it is a conserved amino acid (Figure 2), in which substituted arginine destabilized it.

\section{Discussion}

The GJB2 gene was the first known cause of deafness in the world and Iran [7, 21]. This gene is responsible for about $16-18 \%$ of ARNSHL in Iran. However, there is a large gap in the recognition of other genes responsible [22]. Considering the high frequency of consanguineous marriages in Iran and high prevalence of hearing loss and the heterogeneity of NSHL, many genes are involved $[22,23]$. In this study, targeted genomic capturing was applied to identify an inherited homozygous variation.

The ESRRB gene consists of 11 exons and codes the estrogen-related receptor beta protein, which is associated with hearing loss in mice [24]. ESRRB is a member of orphan nuclear receptors and is related to estrogen receptors (ER), although does not bind to estrogen. The coded protein by ESRRB gene has two important domains including ligand-binding domain (LBD) and DNA-binding domain (DBD) (Figure 3), which are recruited as transcriptional regulation of target genes of estrogen [24]. ESRRB gene is the only known gene that acts as a transcription factor and is associated with ARNSHL, while other transcription factors are related to autosomal dominant NSHL [25].

ESRRB genes are responsible for the recessive DFNB35 deafness forms. The locus DFNB35 has been identified in 2003 by studying a large Pakistani family with NSHL[26], so far more than 12 variations have been identified in DFNB35 which more than half of identified mutations are accrued in Pakistan ethnicity (p.A110V, p.L320P, p.E340del, p.V342L, p.L347P) [24, 27] and the rest of theme belongs to Czech

Republic(p.R291L) [28], Tunisia(p.Y305H) [25], Turkey (p.V342GfsX44) [24] and China (p.R382C) [29] and UAE (c.1058-3 C>A)[30] and our reported variation (p.G167R) Iran population. With exception of c.1156C $>T$ [24] and c.1237G $>A$ [28] which are reported as polymorphism, other variations are considered 
functional mutations (table 2), and most of them are accumulated in the exon 8 ESRRB gene. As shown in figure 3 , the majority of the pathogenic variation occurred in LBD with exception of $p$. A110V and our reported variation in this study, p.Gly167Arg.

In this report, sequence analysis of the ESRRB gene showed c.499G >A mutation as the first variation of ESRRB gene in Iranian ethnicity with ARNSHL and as a very rare cause of ARNSHL in this population which has been submitted by our group with a number rs 1555342141 . c.499G $>A$ is a missense mutation in exon 5 of the ESRRB gene that causes substitution of glycine by arginine in 167 amino acids of ESRRB coded protein. The 167th amino acid is located in the highly conserved domain at DBD and may have deleterious effects on the function of ESRRB. In addition to in silico analysis of c.499G $>A$ variant that is introduced as a damaging variant, based on ACMG Guidelines [31] This variation can be categorized as pathogenic because it is a novel missense variant (PM5) located in a functional domain (PM1), in addition, it is absent in controls (PM2) and is co-segregating in the pedigree (PP1). Molecular modeling prediction revealed that the substitution of an aspartic acid by a glycine residue leads to a large side chain which may increase inappropriate interaction between residues and it seems possibly decreased stability and disrupted the 3D structure of the DBD, therefore abnormal protein in the cells might result in not function properly. Furthermore, multiple sequence alignment of human ESRRB protein across the other species showed that the downstream residues are conserved. Different clinical symptoms as a result of frame-shift and the missense mutations suggest that the molecular mechanism underlying the hearing loss possibly results from a loss of function mutation. This idea supported by Collin .et al study in which in conditional Esrrb/ mice, malfunction hearing and balance coincide with ion homeostasis and disturbed endolymph production, and it is possibly that ESRRB of human is essential for these processes as well [24]. This protein is considered a key factor for the function and development of the inner ear by mediating the effects of estrogens, glucocorticoid hormone, and thyroid hormone, which all are essential for inner ear cells. Also, it might be crucial for the preservation of hearing with aging in human adults, in other hand an animal study has shown that ESRRB/knock-out mice cause hearing impairment; however, it is not in accordance with the suggested role of estrogen in protecting hearing in DFNB35 patients [24].

\section{Conclusion}

The results of this study revealed variation in ESRRB genes and they have not been reported in the literature before and in any database. Application of fast and cost-effective NGS strategy as a diagnostic tool facilitates identification of the corresponding mutation among hearing loss heterogeneous genes and provides an opportunity to identify the other unknown causatives of $\mathrm{HL}$ gene mutation. Moreover, this report highlights the significance of family history and consanguinity marriage, which is common in Iran and increases the chance of homozygous mutations.

\section{Abbreviations}

ER: Estrogen receptors; HGMD: Human Gene Mutation Database ; NGS: next-generation sequencing; GHL: genetic hearing loss; HL: hearing loss; DBD: DNA-binding domain; ARNSHL: autosomal recessive non- 
syndromic hearing loss; MT-RNR1: mitochondrially encoded 12S RRNA; HHL: hereditary hearing loss; YASARA: yet another scientific artificial reality application; SDM: site directed mutator; MAF: minor allele frequency; LBD: ligand-binding domain. GJB2: gap junction beta-2 protein; GJB6: gap junction beta 6 protein

\section{Declarations}

\section{Ethics approval and consent to participate}

Full informed consent was obtained from the patients and all members of their families which participated in the study. The study and methods used for the investigation involving humans were approved by the ethics committee of Tabriz University of Medical Sciences.A copy of the written consent is available for review by the Editor of this journal.

\section{Consent for publication}

Written informed consent for publication was obtained from the all of the participants and the parents of the minors.

\section{Availability of data and materials}

The datasets generated and/or analysed during the current study have uploaded in the NCBI dbVar repository (https://www.ncbi.nlm.nih.gov/projects/SNP/snp_ss.cgi?subsnp_id=ss2137543931). The accession number for the variation wich submitted in clinvar is SCV001739271 and available at https://www.ncbi.nlm.nih.gov/clinvar/submitters/508148/.

\section{Competing interests}

The authors declare that they have no competing interests.

\section{Funding}

The study was financially supported by Neurosciences Research Center, Tabriz University of Medical Sciences (grant number of 94/2-7/13), and the paper was extracted from the MSc dissertation of Tohid Ghasemnejad.

\section{Authors' contributions}

TG, analyzed and interpreted the patient data,JNN, literature review and drafted the manuscript, MSK,reviewed clinical and laboratory data, SMD,study design and finalized this manuscript. All authors read and approved the final manuscript.

\section{Acknowledgments}

We are so grateful to patients and their respected families who kindly consented to join the study. 


\section{References}

1. Morton, C.C. and W.E. Nance. Newborn hearing screening-a silent revolution. New Engl. J. Med. 2006; 354(20):2151-2164.

2. Van Eyken, E., G. Van Camp, and L. Van Laer. The complexity of age-related hearing impairment: contributing environmental and genetic factors. Audiology and Neurotology 2007; 12(6):345-358.

3. Ouyang, X.M., et al. The genetic bases for non-syndromic hearing loss among Chinese. J. Hum. Genet. 2009; 54(3):131-140.

4. Tekin, M., K.S. Arnos, and A. Pandya. Advances in hereditary deafness. The Lancet 2001; 358(9287):1082-1090.

5. Shearer, A.E., et al. Utilizing ethnic-specific differences in minor allele frequency to recategorize reported pathogenic deafness variants. The American Journal of Human Genetics 2014; 95(4):445453.

6. Tekin, M., et al. Moderate hearing loss and pseudodominant inheritance due to L90P/35delG mutations in the GJB2 (connexin 26) gene. Genetic counseling (Geneva, Switzerland) 2003; 14(4):379-386.

7. Babanejad, M., et al. A comprehensive study to determine heterogeneity of autosomal recessive nonsyndromic hearing loss in Iran. Am. J. Med. Genet. A 2012; 158(10):2485-2492.

8. Yazdanpanahi, N., et al. Two novel SLC26A4 mutations in Iranian families with autosomal recessive hearing loss. Int. J. Pediatr. Otorhinolaryngol. 2012; 76(6):845-850.

9. Fattahi, Z., et al. Screening for MY015A gene mutations in autosomal recessive nonsyndromic, GJB2 negative Iranian deaf population. Am. J. Med. Genet. A 2012; 158(8):1857-1864.

10. Sloan-Heggen, C.M., et al. Characterising the spectrum of autosomal recessive hereditary hearing loss in Iran. J. Med. Genet. 2015; 52(12):823-829.

11. Alasti, F., et al. A novel TECTA mutation confirms the recognizable phenotype among autosomal recessive hearing impairment families. Int. J. Pediatr. Otorhinolaryngol. 2008; 72(2):249-255.

12. Hashemzadeh Chaleshtori, M., et al. Novel mutations in the pejvakin gene are associated with autosomal recessive non-syndromic hearing loss in Iranian families. Clin. Genet. 2007; 72(3):261263.

13. Davoudi-Dehaghani, E., et al. A transversion mutation in non-coding exon 3 of the TMC1 gene in two ethnically related Iranian deaf families from different geographical regions; evidence for founder effect. Int. J. Pediatr. Otorhinolaryngol. 2013; 77(5):821-826.

14. Taghizadeh, S.H., et al. Investigation of LRTOMT gene (locus DFNB63) mutations in Iranian patients with autosomal recessive non-syndromic hearing loss. International journal of molecular and cellular medicine 2013; 2(1):41.

15. Mehrjoo, Z., et al. Two novel mutations in ILDR1 gene cause autosomal recessive nonsyndromic hearing loss in consanguineous Iranian families. Journal of genetics 2015; 94(3):483-487. 
16. Grillet, N., et al. Mutations in LOXHD1, an evolutionarily conserved stereociliary protein, disrupt hair cell function in mice and cause progressive hearing loss in humans. The American Journal of Human Genetics 2009; 85(3):328-337.

17. Chen, W., et al. Mutation of COL11A2 causes autosomal recessive non-syndromic hearing loss at the DFNB53 locus. J. Med. Genet. 2005; 42(10):e61-e61.

18. Shearer, A.E., et al. Utilizing ethnic-specific differences in minor allele frequency to recategorize reported pathogenic deafness variants. The American Journal of Human Genetics 2014; 95(4):445453.

19. Ołdak, M., Next Generation Sequencing in Vision and Hearing Impairment, in Clinical Applications for Next-Generation Sequencing. 2016, Elsevier. p. 153-170.

20. Moteki, H., et al. Comprehensive genetic testing with ethnic-specific filtering by allele frequency in a Japanese hearing-loss population. Clin. Genet. 2016; 89(4):466-472.

21. Kenneson, A., K.V.N. Braun, and C. Boyle. GJB2 (connexin 26) variants and nonsyndromic sensorineural hearing loss: a HuGE review. Genet. Med. 2002; 4(4):258-274.

22. Ghasemnejad, T., et al. An update of common autosomal recessive non-syndromic hearing loss genes in Iranian population. Int. J. Pediatr. Otorhinolaryngol. 2017.

23. Saadat, M., M. Ansari-Lari, and D. Farhud. Short report consanguineous marriage in Iran. Ann. Hum. Biol. 2004; 31(2):263-269.

24. Collin, R.W., et al. Mutations of ESRRB encoding estrogen-related receptor beta cause autosomalrecessive nonsyndromic hearing impairment DFNB35. The American Journal of Human Genetics 2008; 82(1):125-138.

25. Saïd, M.B., et al. A novel missense mutation in the ESRRB gene causes DFNB35 hearing loss in a Tunisian family. Eur. J. Med. Genet. 2011; 54(6):e535-e541.

26. Ansar, M., et al. A novel autosomal recessive non-syndromic deafness locus (DFNB35) maps to 14q24. 1-14q24. 3 in large consanguineous kindred from Pakistan. Eur. J. Hum. Genet. 2003; 11(1):77-80.

27. Lee, $\mathrm{K}$., et al. A novel ESRRB deletion is a rare cause of autosomal recessive nonsyndromic hearing impairment among Pakistani families. Genet. Res. Int. 2011; 2011.

28. Brožková, D.Š., et al. DFNB35 due to a novel mutation in the ESRRB gene in a Czech consanguineous family. Int. J. Pediatr. Otorhinolaryngol. 2012; 76(11):1681-1684.

29. Yang, T., et al. Genetic etiology study of the non-syndromic deafness in Chinese Hans by targeted next-generation sequencing. Orphanet J. Rare Dis. 2013; 8(1):85.

30. Chouchen, J. and A. Tlili. Two new mutations, ESPN c. 2257T > C and ESRRB c. 10583 C > A, cause hearing loss in UAE families. Hamdan Medical Journal 2020; 13(2):115.

31. Richards, S., et al. Standards and guidelines for the interpretation of sequence variants: a joint consensus recommendation of the American College of Medical Genetics and Genomics and the Association for Molecular Pathology. Genet. Med. 2015; 17(5):405. 
Figures

A

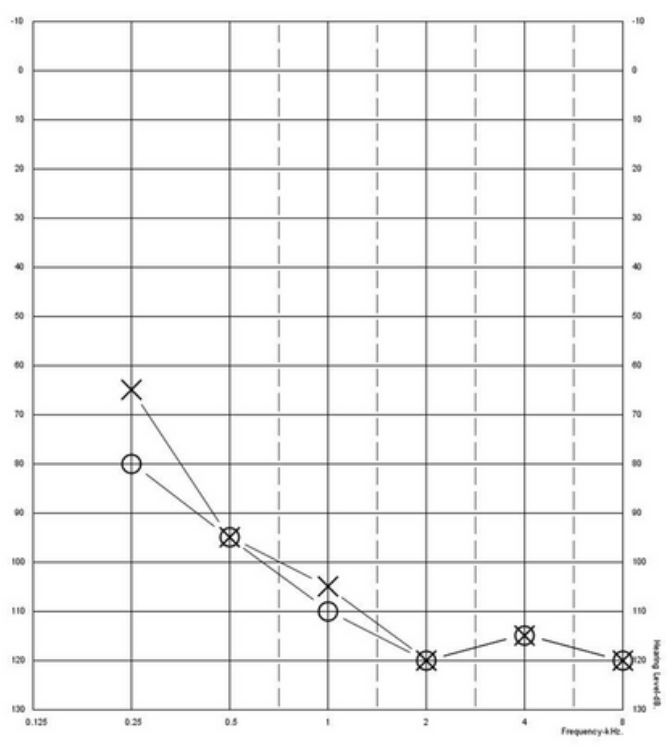

I
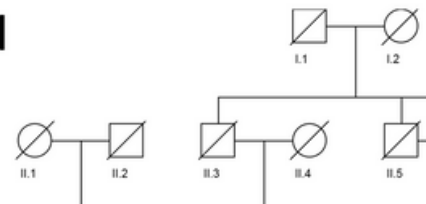

$\bigsqcup_{n \rightarrow 3} \varnothing_{n 4}$
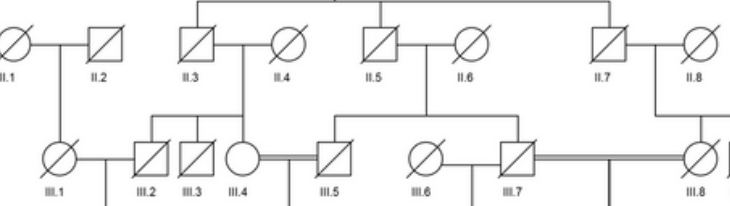

N.1

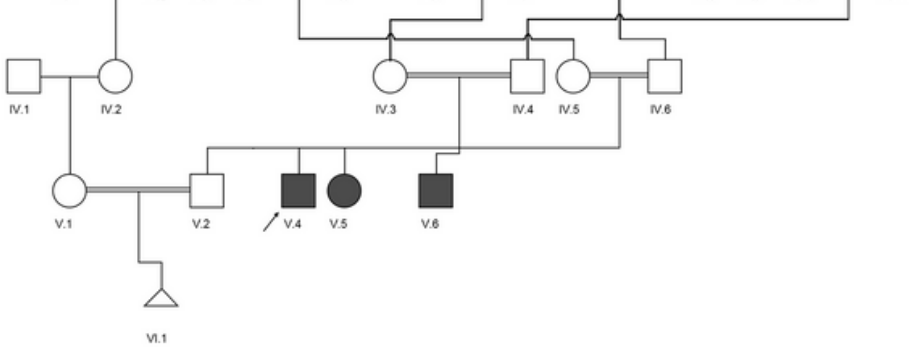

C a

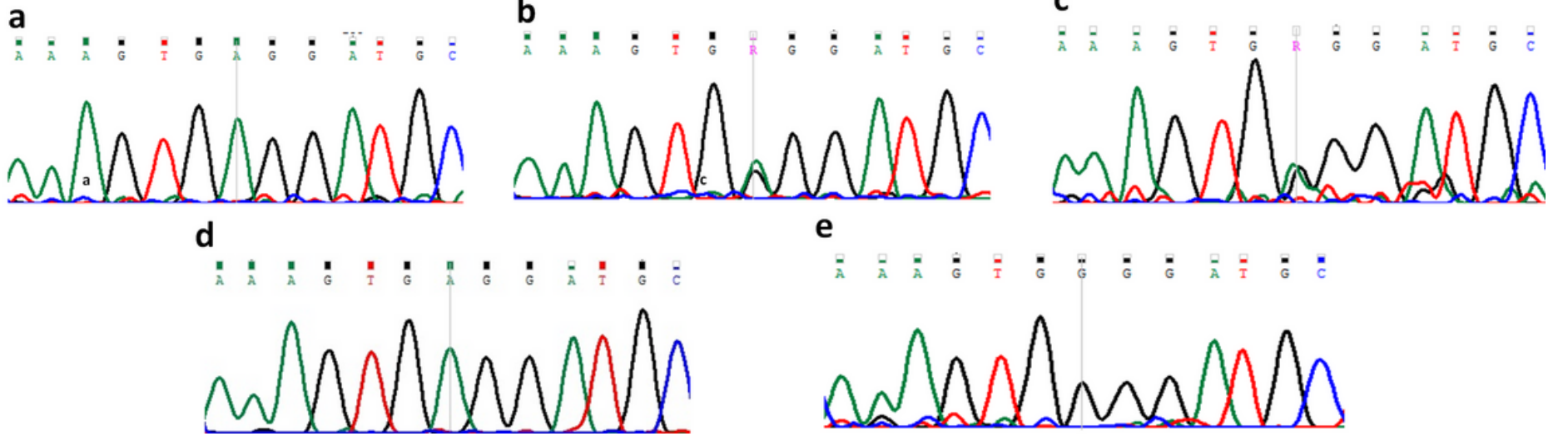

Figure 1

Audiogram, pedigree, and chromatograms of family. (A) The audiogram shows bilateral severe to profound hearing loss in V.4; (B) Pedigree shows a consanguineous marriage between proband parents lead to inheritance autosomal recessive non-syndromic hearing loss; (C)Sequence chromatograms for (a) proband (V.4) (b) father (IV.6) (c) mother (IV.5) and sibling which including (d) affected sister (V.5) and (e) unaffected brother (V.2) 


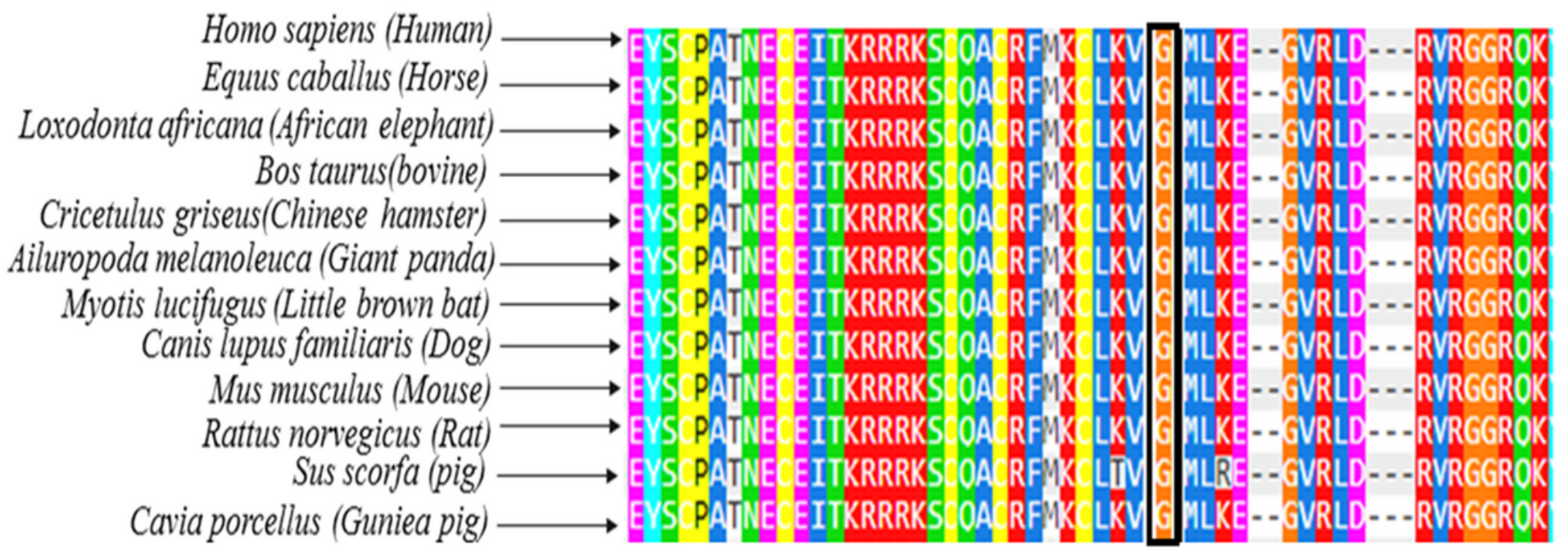
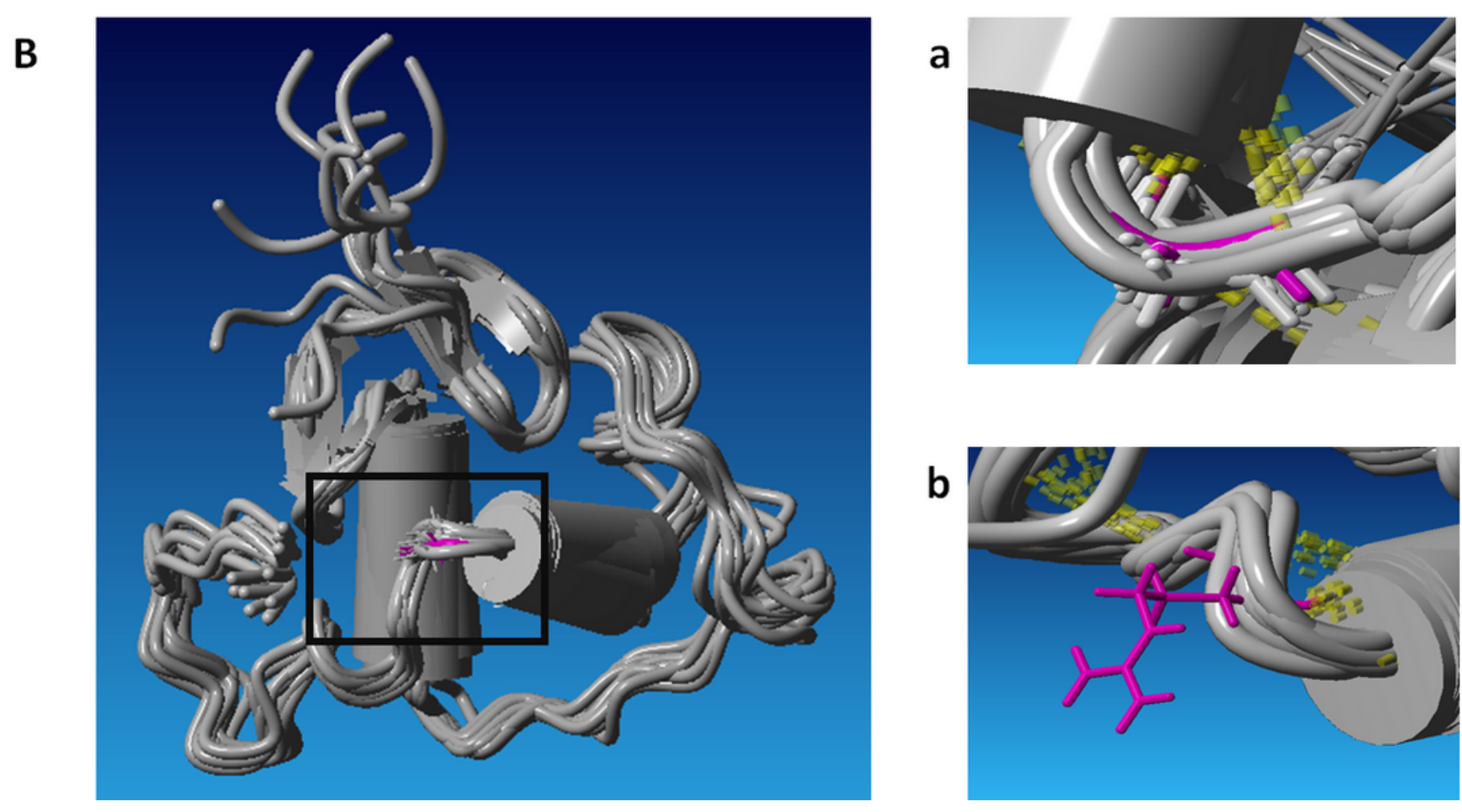

\section{Figure 2}

(A) Visualization of multiple sequence alignment of ESRRB protein in mammals including the missense mutation site. The locations of changed amino acids are indicated with a box. (B) Molecular model of ESRRB protein. The wild-type residue (Gly167) of ESRRB protein is depicted with violet. (a) Molecular modeling predicted hydrogen interaction between Gly167 and Ala163 residues. Hydrogen bonds are shown with the yellow dotted line. (b) the large side chain of Arg in the 167th position possibly increases inappropriate interaction between residues. 

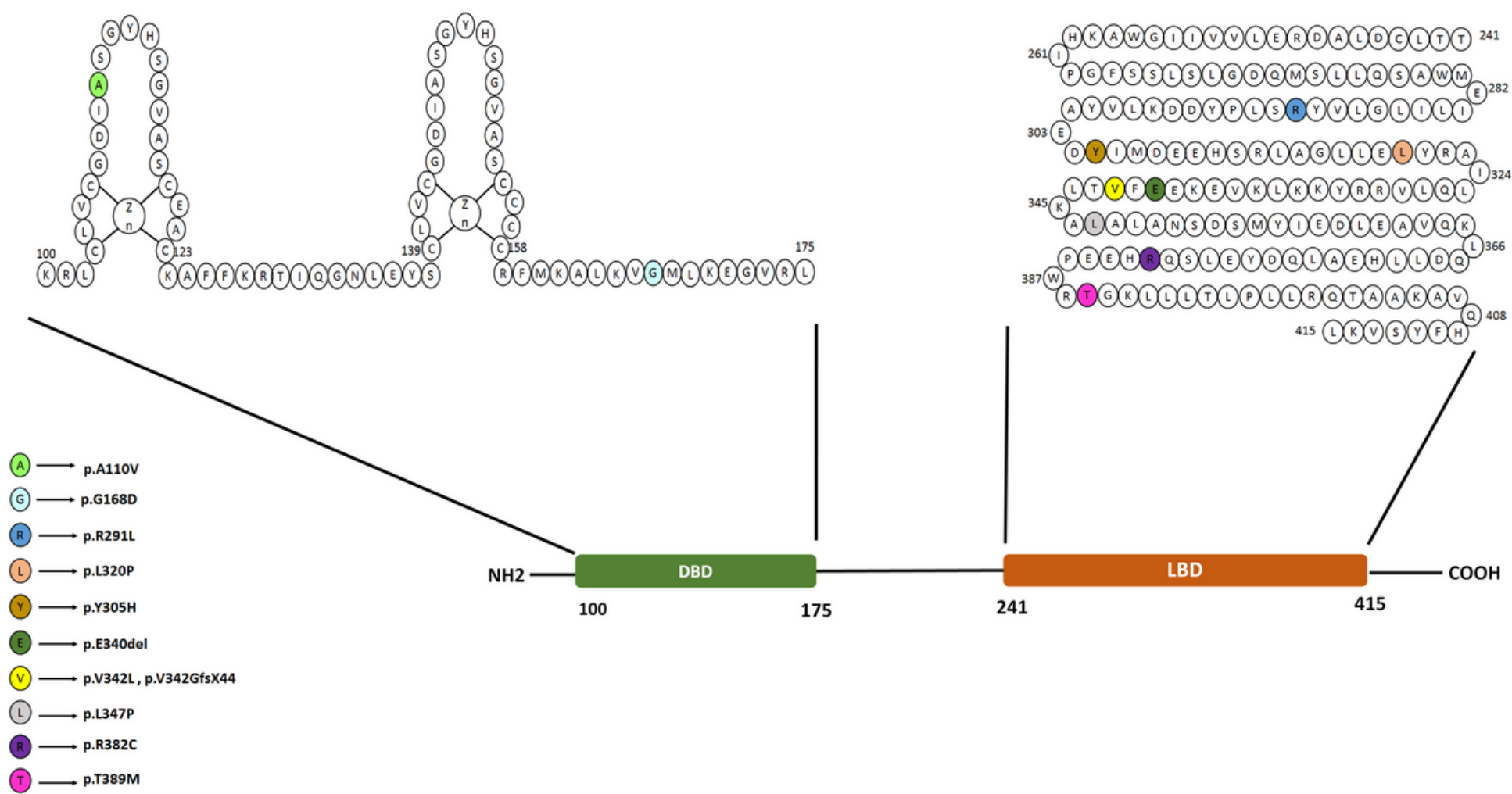

Figure 3

Structure, domains, and mutations in ESRRB protein reported so far. 